\title{
STOCK EXCHANGES IN INDIA- NEED FOR A PARADIGM SHIFT IN ELECTRONIC ERA
}

\author{
Shacheendran.V \\ Head \& Assistant Professor, Post Graduate Department of Commerce, GPM Govt College \\ Manjeswar, Kasaragod District, Kerala,671323
}

Article DOI: https://doi.org/10.36713/epra4434

\begin{abstract}
Stock Exchange is regarded as an essential concomitant of the capitalistic system of economy. It is indispensable for the proper functioning of corporate enterprise. It is the citadel of capital and the pivot of money market. Since 1887, various regional stock exchanges have been set up in India. However, the performance records from 1993-94 to 2003-04 show that the trading initiated by them had been gradually declining. Hence, the present paper suggests, based on their performance records, to consolidate the secondary market for securities by merging them with the leading exchanges.
\end{abstract}

KEY WORDS: Stock Exchange, money market, liquidity, securities

\section{INTRODUCTION}

Stock exchange is a market in which securities are bought and sold. It is an essential component of a developed capital market. The Securities Contracts (Regulation) Act, 1956, defines stock exchange as an association, organisation or body of individuals whether incorporated or not, established for the purpose of assisting, regulating and controlling of business in buying, selling and dealing in securities". A stock exchange, thus, by imparting marketability and liquidity to the securities, encourage investments in securities and assists corporate growth.

\section{IMPORTANCE OF STOCK EXCHANGE}

- It provides an organised market for transactions in shares \& other securities and thereby facilitates, liquidity/ marketability.

- It promotes the habit of savings and investment among general public by providing a secondary market and thereby helps capital formation.

- It ensures a measure of safety and fair dealing to protect investors' interests.
- It provides an instant valuation of securities caused by changes in the environment.

- Stock exchange is an essential concentant of capitalistic system of economy.

- It induces companies to improve persons as the market price at the stock exchange reflex the performance.

- It promotes industrial growth and industrial investments.

\section{STOCK EXCHANGES IN INDIA - HISTORY IN BRIEF}

The Indian stock market is one of the oldest in Asia. The earliest records of security dealings in India are meagre and obscure. The East India company was the dominant institution in those days and business in its loan securities was transacted towards the close of the eighteenth century.

By the 1830's business in corporate stocks and shares in bank and cotton presses took place in Bombay (Now Mumbai). Though the trading list was broader in 1839, there were only half a dozen brokers recognised by banks and merchant.

In 1860-61, the American Civil War broke out and cotton supply from the United States of 


\section{EPRA International Journal of Research and Development (IJRD)}

Volume: 5 | Issue: 5 | May 2020

- Peer Reviewed Journal

America (USA) and Europe was stopped. This resulted in the 'sharemania' for cotton trading in India. The number of brokers increased to 250 . However, at the end of the American Civil War, in 1865, a disastrous slump began. The share prices plummeted. At the sametime, brokers found a place in Dalal Street, Bombay, where they could conveniently assemble and transact business. In 1887, they formally established the "Native Share and Stock Brokers' Association". In 1895, the Association acquired premises in the same street. It was inaugurated in 1899 as the Bombay Stock
Exchange (BSE). In November 1992 National Stock Exchange of India Limited (NSE) was set up, promoted by leading financial institutions at the behest of the Government of India. In 1990, the Over the Counter Exchange of India (OTCEI) was incorporated with an aim to aid enterprising promoter in raising finance for new projects in a cost effective manner Following this, the various regional exchanges were also set up.

The number of regional exchange in each of the four zones - east, west, north and south - is shown in table 1.1.

Table 1.1.Regional Stock Exchange

\begin{tabular}{|l|l|l|l|}
\hline \multicolumn{1}{|c|}{ North Zone } & \multicolumn{1}{c|}{ East Zone } & \multicolumn{1}{c|}{ West Zone } & \multicolumn{1}{c|}{ South Zone } \\
\hline Kanpur & Bhubaneswar & Ahmedabad & Bangalore \\
\hline Ludhiana & Kolkatha & Vadodara & Chennai \\
\hline New Delhi & Guwahati & Indore & Kochi \\
\hline Jaipur & & Bombay & Coimbatore \\
\hline & & Pune & Hyderabad \\
\hline & & Rajkot & Mangalore \\
\hline
\end{tabular}

Source: SEBI Bulletins.

As of 2003-04, there are 23 stock exchange in India. They are;

1. Ahmedabad Stock Exchange Association

3. Bhubaneswar Stock Exchange

5. Cochin Stock Exchange Ltd.

7. Delhi Stock Exchange Association

9. Hyderabad Stock Exchange Ltd.

11. Ludhiana Stock Exchange Association

14. Madhyapradesh Stock Exchange Ltd.

16. Mumbai Stock Exchange

18. Uttarpradesh Stock Exchange Ltd.

20. Meerut Stock Exchange Ltd.

22. Saurashtra Kutch Stock Exchange Ltd.

Stock Exchange are governed by the Securities Controls (Regulation) Act, 1956. Securities and Exchange Board of India (SEBI) monitors their working.

\section{NEED FOR CONSOLIDATION: FINDINGS, SUGGESTIONS}

Until the introduction of electronic trading, it was necessary to have various regional stock exchanges covering all parts of the country. They followed their own rules. They provided access to deal in securities. However, in the electronic era, the concept of regional exchange are no longer relevant. Online trading and depository services offer better quality and convenient services for the investors from any part of the country. In addition, the regional stock exchanges have been displaying a dismal performance over the period 1992-93 to 2003-04 as depicted in table 1.2.(See Appendix)

The table 1.2. (See Appendix) shows the turnover recorded on cash segments of exchanges in India from the year 1992-93 to 2003-04. It shows that the turnover on cash segments of all the
2.Bangalore Stock Exchange
4.Calcutta Stock Exchange
6.Coimbatore Stock Exchange
8.Guwahati Stock Exchange Ltd.
10.Inter-connected Stock Exchange
13.Madras Stock Exchange
15.Manglore Stock Exchange
17.Pune Stock Exchange
19.OTCEI
21.National Stock Exchange Ltd.
23. Vadodara Stock Exchange Ltd.

regional exchanges have reduced substantaly. Turnover of stock exchange in Bangalore, Bhubaneswar, Cochin, Coimbatore, Guuhati, Jaipur, Ludhiana, Madhya Pradesh, Magadh, Mangalore, Pune \& Vaodara have plummeted to zero by the year 2003-2004; other regional exchange also have been recording falling turnover. The table shows that the Uttarpradesh stock exchange, which recorded the largest turnover among the regional exchanges amounted only to $1.1 \%$ of the turnover of NSE, Mumbai.

Table 1.3 (See Appendix) shows the quantity of shares traded and delivered and their value. It shows that regional exchanges in India deals only nominal portion of share trading \& delivery in India. No regional exchange deals more than $5 \%$ of the total. Among them, the trading has been substantially reduced. For instance, Calcutta Stock Exchange which traded 19548 lakhs shares in 200102 , which was $3.9 \%$ of the total trading in India, recorded only $0.39 \%$ trading in 2003-2004. The results show that there are stock exchanges which deal virtually insignificant trading in their bourses. 


\section{EPRA International Journal of Research and Development (IJRD)}

\section{CONCLUSION}

In earlier centuries India had a few reasons (such as widely scattered investors, regional differences etc.) for setting up of regional stock exchanges in various parts of the country. However, after the launching of electronic trading and depository services, they make it totally irrelevant to have regional exchanges. Investors from any part of the country can very well access the NSE/BSE from anywhere, even from the remotest village. Technological advancement also enables to have transparent, hasslefree and convenient mode of trading and settlement. Unlike paper form based trading, in screen based trading the price at which trade was executed and settled can very well be known to the investors. Hence, it is suggested that the regional exchange should be merged with the NSE / BSE. They may operate like a depository participant (DP) and trading member in NSE/BSE. Necessary legal amendments should be made for the purpose. Such a consolidation will indeed add strength to the efficiency of Indian capital market.

In the electronic era, the depository system has redefined the way of dealing in share market. Hence the conventional stock exchanges have no significant role to play. Having NSE and BSE as the central exchanges are well competent to lead share trading in India, other Stock Exchanges may either be closed down or merge with the big ones. Indeed such a consolidation will help the regulator too for carrying out its functions.

\section{REFERENCES}

1. Batra,G.S. Management of Financial Institutions And Markets, Deep And Deep Publications Pvt. Ltd. Delhi 1997.

2. Bhole, L.M., "The Indian Capital Market At Cross Roads,", Vikalpa, Vol.20, No.2, 29-41. 1995

3. Doughall, H,E, And H.E Gaumitz, Capital Markets And Institutions, Prentice Hall, New Jersey.

4. Edmnister. R.O, Financial Institutions, Markets And Management, Mc-Graw-Hill, New York.

5. Fishcher, Stantley, "Finance System Soundness", Finance And Development, March 1997.

6. Gupta L.C "Challenges Before Securities And Exchange Board Of India”, Economic And Political Weekly, March 23, 1996.

7. Henning And Charles, Financial Markets And The Economy, William Pigotte and et al, Prentice Hall Publications.

8. Jorion, P. And Goetzmann, W.N., Global state Market in The Twentieth Century, The Journal of Finane, No.3, 1999.

9. Kohn, Meir, Financial Institutions And Market, Tata Mc-Graw Hill Publishing Company, Delhi, 1996.
10. Mehta R.C., Capital Markets In India For Planned Growth, Kitab Ghar, Gwalior, 1965,

11. Obaidullah M. "Indian Stock market - Theories And Evidence, The Institute Of Chartered Financial Analysts Of India, 1994.

12. Ojha P.D "Capital Market - Emerging Trends" RBI Bulletin, January 1988.

13. Sahoo M.S. "Securities Market Reforms In A Developing Country", The Chartered Secretary, Nov 308-16, 1977.

14. Singhvi, L.K, "The Future of India's Stock Market - Surveillance Challenges And Reality," 1998.

15. www.sebi.gov.in

16. Capital Market, various issues

17. Dalal Street, various issues 
SJIF Impact Factor: 7.001| ISI I.F.Value:1.241| Journal DOI: 10.36713/epra2016

ISSN: 2455-7838(Online)

\section{EPRA International Journal of Research and Development (IJRD)}

Volume: 5 | Issue: 5 | May 2020

- Peer Reviewed Journal

\section{APPENDIX}

Table 1.2. Distribution of Turnover on Cash Segments of Exchanges

\begin{tabular}{|c|c|c|c|c|c|c|c|c|c|c|c|c|}
\hline Stock Exchange & $1992-93$ & 1993-94 & 1994-95 & 1995-96 & 1996-97 & $1997-98$ & 1998-99 & 1999-00 & 2000-01 & 2001-02 & 2002-03 & 2003-04 \\
\hline Ahmedabad & 22183 & 23540 & 12452 & 20626 & 41065 & 31117 & 29928 & 37566 & 54036 & 14644 & 15459 & 4544 \\
\hline Bangalore & 730 & 2316 & 712 & 897 & 4389 & 8637 & 7749 & 1115 & 600 & 70 & 0 & 0 \\
\hline Bhubaneswar & 1899 & 814 & 303 & 211 & 231 & 203 & 74 & 68 & 0 & 0 & 0 & 0 \\
\hline Calcutta & NA & 57641 & 52822 & 62149 & 105481 & 178779 & 172818 & 357167 & 355035 & 27075 & 6523 & 1928 \\
\hline Cochin & 65 & 382 & 614 & 287 & 152 & 164 & 96 & 66 & 26 & 2 & 0 & 0 \\
\hline Coimbatoe & 27 & 2082 & 3192 & 5007 & 4798 & 4274 & 769 & 78 & 0 & 0 & 0 & 0 \\
\hline Delhi & 7413 & 12099 & 9144 & 10083 & 48992 & 67936 & 50651 & 94528 & 82997 & 5526 & 11 & 3 \\
\hline Gauhati & 443 & 452 & 285 & 616 & 484 & 120 & 52 & 0 & 0 & 0 & 0 & 0 \\
\hline Hyderabad & 676 & 984 & 1160 & 1107 & 480 & 1868 & 1270 & 1236 & 978 & 41 & 5 & 2 \\
\hline ICSE & NA & NA & NA & NA & NA & NA & NA & 274 & 237 & 70 & 53 & 0 \\
\hline Jaipur & 296 & 616 & 879 & 1048 & 1519 & 453 & 63 & 2 & 0 & 0 & 0 & 0 \\
\hline Ludhiana & 1050 & 3150 & 4975 & 4849 & 5274 & 8316 & 6070 & 6872 & 9184 & 964 & 0 & 0 \\
\hline Madhyapradesh & 356 & 132 & 118 & 202 & 5 & 1 & 1 & 10 & 2 & 1 & 0 & 0 \\
\hline Madras & 3407 & 4618 & 6117 & 3259 & 3912 & 2458 & 739 & 500 & 218 & 48 & 76 & 101 \\
\hline Magadh(Patna) & NA & 1938 & 797 & 1629 & 2755 & 323 & 1 & 9 & 2 & 0 & 0 & 0 \\
\hline Mangalore & 12 & 108 & 62 & 39 & 380 & 314 & 11 & 0 & 0 & 0 & 0 & 0 \\
\hline Mumbai & 49696 & 84536 & 67749 & 50064 & 124190 & 207112 & 310750 & 686428 & 1000032 & 307292 & 314073 & 503053 \\
\hline NSE & NA & NA & 1805 & 67287 & 295403 & 370193 & 414474 & 839052 & 1339510 & 513167 & 617989 & 1099534 \\
\hline OTCEI & 2 & 38 & 365 & 224 & 219 & 283 & 198 & 3603 & 126 & 4 & 0 & 16 \\
\hline Pune & 782 & 3459 & 3672 & 7096 & 10084 & 8624 & 4827 & 6090 & 6171 & 1171 & 0 & 0 \\
\hline SKSE & 265 & 614 & 329 & 452 & 395 & 17 & 0 & 0 & 0 & 0 & 0 & 0 \\
\hline Uttarpradesh & 5508 & 6789 & 7923 & 12373 & 16070 & 15209 & 18429 & 23876 & 25112 & 13349 & 14767 & 11751 \\
\hline Vadodara & 1681 & 3751 & 3855 & 2519 & 4344 & 4577 & 1749 & 159 & 2 & 20 & 3 & 0 \\
\hline
\end{tabular}

NA : Not Available,

Source: Various Exchanges 


\section{EPRA International Journal of Research and Development (IJRD)}

Volume: 5 | Issue: 5 | May 2020

- Peer Reviewed Journal

Table 1.3. Trading Statistics on Stock Exchanges

\begin{tabular}{|c|c|c|c|c|c|c|c|c|c|}
\hline \multirow[t]{2}{*}{$\begin{array}{c}\text { Stock } \\
\text { Exchanges }\end{array}$} & \multicolumn{3}{|c|}{$\begin{array}{l}\text { Shares Traded } \\
\text { (Lakh) }\end{array}$} & \multicolumn{3}{|c|}{$\begin{array}{l}\text { Shares Deliverd } \\
\text { (Lakh) }\end{array}$} & \multicolumn{3}{|c|}{$\begin{array}{l}\text { Value of Shares Delivered } \\
\text { (Rs.crore) }\end{array}$} \\
\hline & 2001-02 & 2002-03 & 2003-04 & $\begin{array}{l}\text { 2001- } \\
02\end{array}$ & $\begin{array}{c}2002- \\
03\end{array}$ & $\begin{array}{c}2003- \\
04\end{array}$ & $\begin{array}{l}\text { 2001- } \\
02\end{array}$ & $\begin{array}{c}2002- \\
03\end{array}$ & 2003-04 \\
\hline Ahmedabad & $\begin{array}{l}7622 \\
(1.5)\end{array}$ & 7239 (1.20) & $\begin{array}{l}2514 \\
(0.23)\end{array}$ & $373(0.3)$ & $115(0.10)$ & $115(0.04)$ & $435(0.04)$ & 99 & 0 \\
\hline BSE & $\begin{array}{c}182196 \\
(36.1)\end{array}$ & $\begin{array}{c}221403 \\
(3.64)\end{array}$ & $\begin{array}{l}388748 \\
(34.84)\end{array}$ & $\begin{array}{l}57668 \\
(46.9)\end{array}$ & $\begin{array}{c}71131 \\
(46.00)\end{array}$ & $\begin{array}{l}144531 \\
(45.09)\end{array}$ & $\begin{array}{l}59980 \\
(36.20)\end{array}$ & $\begin{array}{c}50308 \\
(36.95)\end{array}$ & $\begin{array}{l}129312 \\
(36.95)\end{array}$ \\
\hline Bangalore & $\begin{array}{c}35 \\
(0.01)\end{array}$ & $\begin{array}{c}0.0 \\
(0.00)\end{array}$ & $\begin{array}{c}3 \\
(0.00)\end{array}$ & $\begin{array}{c}8 \\
(0.01)\end{array}$ & $\begin{array}{c}0.0 \\
(0.00)\end{array}$ & $\begin{array}{c}3 \\
(0.00)\end{array}$ & $\begin{array}{c}20 \\
(0.00)\end{array}$ & $\begin{array}{c}0.0 \\
(0.00)\end{array}$ & 0.5 \\
\hline Bhubabeswar & 0 & NA & 0 & 0 & 0 & 0 & 0 & 0 & 0 \\
\hline Calcutta & $\begin{array}{c}19548 \\
(3.9)\end{array}$ & $\begin{array}{l}6837 \\
(1.10)\end{array}$ & $\begin{array}{l}4378 \\
(0.39)\end{array}$ & $\begin{array}{l}2062 \\
(1.7)\end{array}$ & $\begin{array}{c}904 \\
(0.60)\end{array}$ & $\begin{array}{c}1225 \\
(0.60)\end{array}$ & $\begin{array}{c}1810 \\
(0.30)\end{array}$ & $\begin{array}{c}400 \\
(0.08)\end{array}$ & 266 \\
\hline Cochin & 29 & 0 & 0 & 0.8 & 0 & 0 & 2 & 0 & 0 \\
\hline Coimbatore & 0 & 0 & 0 & 0 & 0 & 0 & 0 & 0 & 0 \\
\hline Delhi & $\begin{array}{l}6811 \\
(1.4)\end{array}$ & $\begin{array}{c}34 \\
(0.01)\end{array}$ & $\begin{array}{c}0.03 \\
(0.00)\end{array}$ & $\begin{array}{l}3127 \\
(2.5)\end{array}$ & $\begin{array}{c}22 \\
(0.00)\end{array}$ & $\begin{array}{c}0.03 \\
(0.00)\end{array}$ & $\begin{array}{c}1872 \\
(0.00)\end{array}$ & $\begin{array}{c}2 \\
(0.00)\end{array}$ & 0.14 \\
\hline Gauhati & $0.13(0.0)$ & $0.21(0.0)$ & 0 & 0.13 & 0.05 & 0 & 0.03 & 0.21 & 0 \\
\hline Hyderabad & $\begin{array}{c}143 \\
(0.03)\end{array}$ & $\begin{array}{c}37 \\
(0.01)\end{array}$ & $\begin{array}{c}17 \\
(0.00)\end{array}$ & $\begin{array}{c}82 \\
(0.07)\end{array}$ & $\begin{array}{c}14 \\
(0.01)\end{array}$ & $\begin{array}{c}17 \\
(0.01)\end{array}$ & $\begin{array}{c}8 \\
(0.00)\end{array}$ & $\begin{array}{c}2 \\
(0.00)\end{array}$ & 2 \\
\hline ICSE & $\begin{array}{c}123 \\
(0.02)\end{array}$ & $\begin{array}{c}30 \\
(0.01)\end{array}$ & $\begin{array}{c}0.06 \\
(0.00)\end{array}$ & $\begin{array}{c}0.65 \\
(0.00)\end{array}$ & $\begin{array}{c}0.1 \\
(0.00)\end{array}$ & $\begin{array}{c}10 \\
(0.00)\end{array}$ & $\begin{array}{c}0.1 \\
(0.00)\end{array}$ & $\begin{array}{c}0.1 \\
(0.00)\end{array}$ & 0.03 \\
\hline Jaipur & 0 & 0 & 0 & 0 & 0 & 0 & 0 & 0 & 0 \\
\hline Ludhiana & $765(.20)$ & $0(0.1)$ & 0 & 74 & 0 & 0 & 53 & 0 & 0 \\
\hline Madras & $52(0.01)$ & $0(.01)$ & $85(0.004)$ & $5(0.00)$ & $0(0.00)$ & 0.96 & 2.19 & 0 & 0.19 \\
\hline Magadh & $0(0.00)$ & $0.11(0.00)$ & 0.19 & 0 & 0 & 0 & 0 & 0 & 0 \\
\hline Mangalore & 0 & 0 & 0 & 0 & 0 & 0 & 0 & 0 & 0 \\
\hline MPSE & $11(0.0)$ & 0 & 0 & 0.70 & 0 & 0 & 0.9 & 0 & 0 \\
\hline NSE & $\begin{array}{c}278409 \\
(55.2)\end{array}$ & $\begin{array}{c}364066 \\
(59.8)\end{array}$ & $\begin{array}{c}713301 \\
(48.2)\end{array}$ & $\begin{array}{c}82353 \\
(53.20)\end{array}$ & $\begin{array}{l}174538 \\
(55.460\end{array}$ & $\begin{array}{c}71765 \\
(63.46)\end{array}$ & $\begin{array}{c}71765 \\
(63.30)\end{array}$ & $\begin{array}{l}87956 \\
(62.95)\end{array}$ & $\begin{array}{c}220341 \\
(62.6)\end{array}$ \\
\hline OTCEI & $\begin{array}{c}5.41 \\
(0.001)\end{array}$ & $\begin{array}{c}1.8 \\
(0.00)\end{array}$ & $\begin{array}{l}22.21 \\
(0.00)\end{array}$ & $\begin{array}{l}0.07 \\
(0.0)\end{array}$ & 0 & 0 & 0.03 & 0 & 0 \\
\hline Pune & $396(0.1)$ & $0.5(0.01)$ & 0.00 & 16 & 0.03 & Nil & 34 & 0.01 & Nil \\
\hline SKSE & 0 & 0 & 0 & - & 0 & Nil & 0 & 0 & Nil \\
\hline UPSE & $\begin{array}{l}7997 \\
(1.6)\end{array}$ & $\begin{array}{l}9288 \\
(1.50)\end{array}$ & $\begin{array}{l}6737 \\
(0.60)\end{array}$ & $\begin{array}{c}202 \\
(0.2)\end{array}$ & $\begin{array}{c}235 \\
(0.20)\end{array}$ & $\begin{array}{c}74 \\
(0.02)\end{array}$ & $\begin{array}{c}232 \\
(0.20)\end{array}$ & $\begin{array}{c}230 \\
(0.02)\end{array}$ & 76 \\
\hline Vadodaa & $\begin{array}{c}7.0 \\
(001)\end{array}$ & $\begin{array}{c}8 \\
(0.00)\end{array}$ & $\begin{array}{c}0 \\
(0.00)\end{array}$ & 0 & 0 & Nil & 0 & 0 & Nil \\
\hline Total & $5,04,149$ & $60,89,44$ & $11,15,807$ & $1,22,917$ & $1,54,775$ & $3,20,504$ & $1,36,225$ & $1,38,997$ & $3,49,997$ \\
\hline
\end{tabular}

Figures in parentheses indicate percentage to total

Source: various Stock Exchange 